\title{
Targeted conditional gene knockout in human embryonic stem cells
}

Cell Research (2010) 20:379-382. doi: 10.1038/cr.2010.23; published online 9 February 2010

\section{Dear Editor,}

We report our application of a promoterless knockout (KO) strategy combined with Flpase- and Cre-mediated recombination to efficiently ablate gene function in human embryonic stem (hES) cells. Using this strategy, we modified and deleted both alleles of BAF250a (ARID1A), a signature component of the ATP-dependent SWI/ SNF chromatin remodeling complex BAF. This strategy should facilitate the functional studies of genes essential for hES cell self-renewal and differentiation, and be instrumental for human therapeutic applications.

The past two decades have witnessed the revolution in studying mammalian gene functions, with the development of gene KO technology in mouse [1]. Typically, homologous recombination-mediated gene DNA sequence replacement creates heterozygous (Het) mouse ES cells of a gene of interest. These ES cells are then injected into mouse blastocysts to generate Het animals. Homozygous $\mathrm{KO}$ (null) mice can then be generated with the intercross of Het animals. Null ES cell lines are normally derived from null blastocysts, but can be obtained with sequential targeting of two $\mathrm{KO}$ vectors bearing different selection markers [2] or with a high dose of drug selection that potentially leads to substantial modification in the genome [3]. However, the generation of hES cell lines bearing gene-specific KO with homologous recombination has proved to be very difficult due to challenges in hES cell culture and the lack of efficient means to transfect and target $\mathrm{KO}$ vectors for biallelic gene inactivation [4].

Consequently, other methods, such as phiC31 integrase-mediated gene insertion [5] and engineered zinc finger nuclease (ZFN)-mediated gene editing [6], have been applied to manipulate gene expression in hES cells, and could be modified to ablate gene expression. In fact, ZFN-mediated gene KOs were accomplished in hES/ iPS cells just recently [7, 8]. However, the efficiency and specificity of ZFN-mediated gene $\mathrm{KO}$ may have to be determined experimentally; the design of ZFN pairs for gene-specific sequences is technically challenging to most research labs and whether researchers are willing to pay the high price for commercial custom-designed ZFNs (Sigma) remains to be seen.

A conditional KO strategy that we applied successfully to ablate BAF250a (ARID1A) function in mouse ES cells [9] and its alternatives could be practical for hES KO studies. This strategy consisted of an entirely in vitro approach in which both copies of the BAF250a gene were targeted sequentially in ES cells using the same targeting vector to avoid the possible lethal phenotype of BAF250a KO ES cells. This in vitro strategy utilized promoterless [10] and reading frame-enhanced homologous recombination to increase targeting efficiency. The initial $\mathrm{KO}$ allele was converted back into a reverted wild type (rWT, f (floxed by two loxP sites)) by Flpase-mediated recombination. The same KO vector was used again to target the second allele of BAF250a. Finally, Cre-mediated recombination renders both copies into mutant alleles lacking a critical exon that leads to open-reading frame shift and nonsense-mediated decay of the mRNA. This strategy effectively eliminates gene function in a highly controlled manner following the activation of Cre-ERT2 [11] with the ligand 4-hydroxy tamoxifen (4-OH TAM).

To determine if this strategy is applicable in ablating genes in hES cells, and if BAF250a is also essential for hES cell pluripotency or maintenance, we attempted to knock out human BAF250a gene using the NIH H9 ES cells (Figure 1A and 1B, see Supplementary information, Data S1 for all the experimental details). A PCR-amplified $0.7-\mathrm{kb}$ fragment of the exon 8 region was inserted in front of the $\beta$-geo cassette in the conditional $\mathrm{KO}$ vector, followed by sequential insertions of PCR-amplified 3.9$\mathrm{kb}$ and $3.7-\mathrm{kb}$ genomic fragments that are $5^{\prime}$ and $3^{\prime}$ to the exon 8 of BAF250a [9]. After NotI digestion, $25 \mu \mathrm{g}$ of the linearized vector was electroporated into $10^{7} \mathrm{ES}$ cells using BioRad gene pulser set at $320 \mathrm{~V}, 200 \mu \mathrm{F}$. ES cells were then plated onto 10 -cm culture dishes $\left(\sim 2 \times 10^{6}\right.$ per dish) with $10 \mathrm{ml}$ of fresh hES medium. After 3 days, 50 $\mu \mathrm{g} / \mathrm{ml} \mathrm{G} 418$ was added to the culture, and ES cells were cultured under G418 selection for 5 days before G418 removal. During the process, the culture medium was changed every day until colonies were ready for picking. 
A

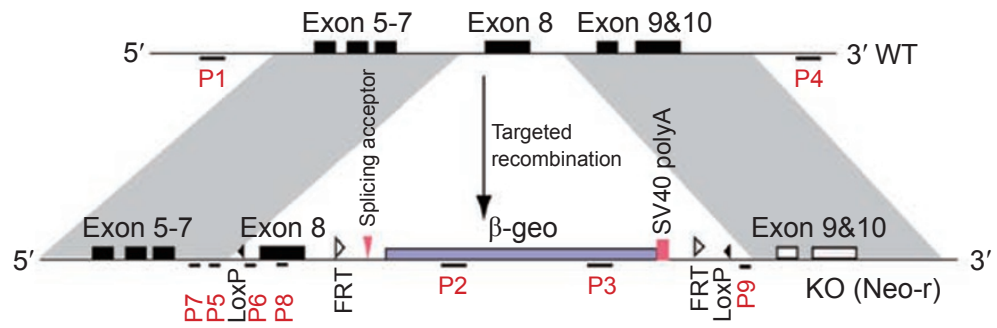

C

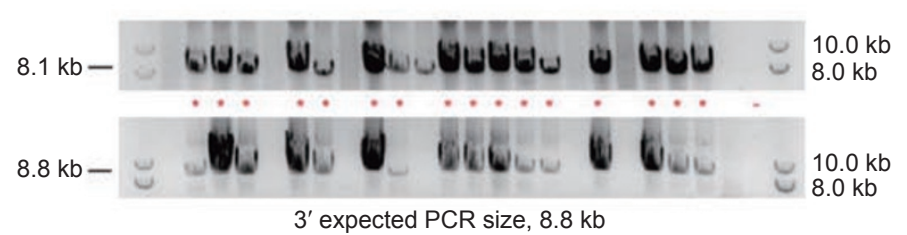

D

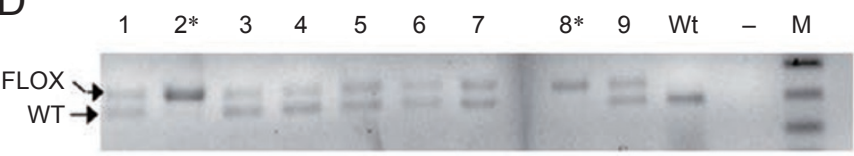

E

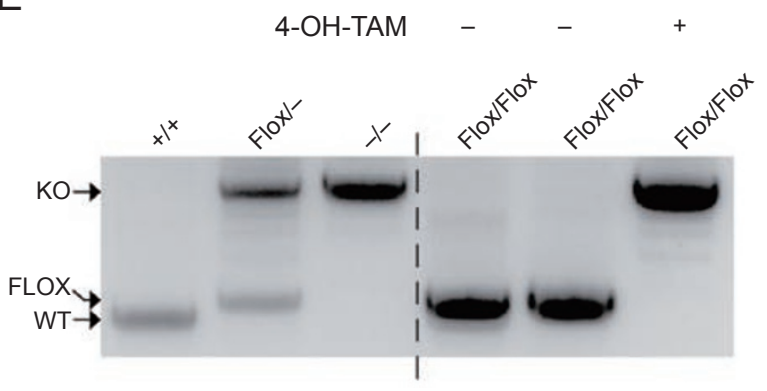

$\mathrm{H}$

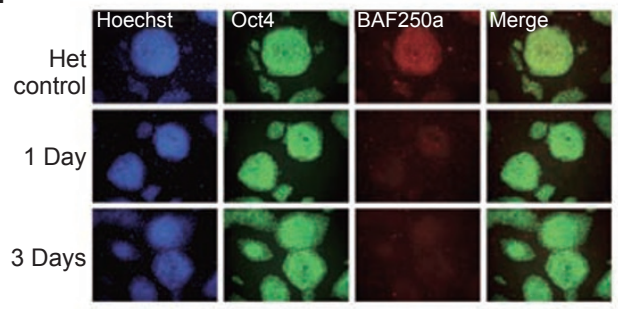

B

(Het)
BAF250a +/f
(WT)
2nd
electroporation
BAF250a neo-r/f (-/f)
(Het)
Cre
BAF250a Neo-s/Neo-s

(KO)

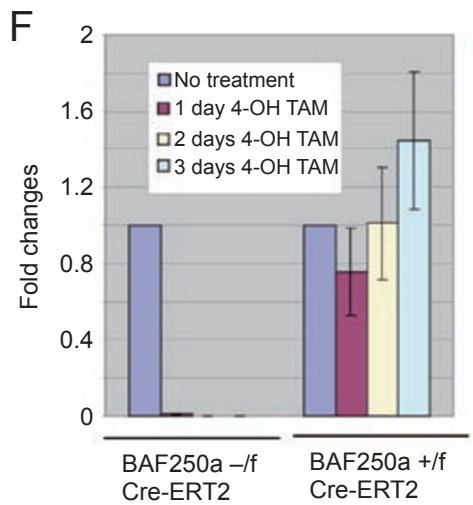

G

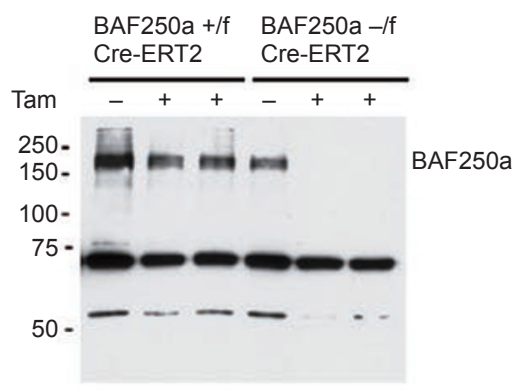

Figure 1 A strategy to generate BAF250a-null human ES cells. (A) Diagram of the strategy for homologous recombination. (B) Derivation of BAF250a homozygous ES cells from WT hES cells. The initial KO allele is neo-resistant and labeled as Neo-r, and the Cre-treated KO alleles at the final step lack exon 8 and are neo-sensitive and labeled as Neo-s. (C) Long-range PCR with primers P1-P4 confirmed homologous recombination. (D) PCR using P5 and P6 confirmed the successful targeting of both BAF250a alleles, as detected by the presence of an extra loxP sequence in front of exon 8 (lanes 2 and 8 ). flox/flox (-/flox): 341 bp; flox/+: 341 bp/290 bp; +/+: 290 bp. (E) PCR using P7-9 identified WT, Flox (rWT) and exon 8 deletion (-) alleles. (F) Time course of mRNA expression after 4-OH TAM treatment for BAF250a f/f; Cre-ERT2 and BAF250 f/+; Cre-ERT2 cell lines. (G) After transfection of a CAG tamoxifen-inducible Cre-ERT2 plasmid into the BAF250a -/f ES cells, 24-h 4-OH TAM treatment completely ablated BAF250a protein expression, as judged by western blotting. $(\mathbf{H})$ Immunostaining of BAF250a, Oct4 and Nanog. BAF250a Het cells were used as controls. f, flox allele (reverted WT allele); P, primer. 
G418-resistant colonies were picked, trypsinized and passaged as described [12].

Long-range PCR was used to identify the initial KO allele generated by homologous recombination between a BAF250a WT allele and the two homologous sequences in the $\mathrm{KO}$ vector. Out of the 22 clones examined, 16 were positively targeted BAF250a Het hES cell lines (Figure 1C), an efficiency comparable to what was observed in mouse ES cells [9]. These ES cells are LacZ positive and appear morphologically normal (data not shown).

To avoid the possibility that complete ablation of BAF250a causes lethality of hES cells, two such Het cell lines were then transiently transfected with $2 \mu \mathrm{g}$ of a nonlinearized CAG-Flpase plasmid (containing a puromycin cassette) to revert the initial KO allele back to a WT (rWT, f) allele (Figure 1B). Two days after electroporation, 0.5 $\mu \mathrm{g} / \mathrm{ml}$ puromycin was added to the medium for 2 days. Cells were then cultured without the drug until colonies were formed and ready for picking. Picked colonies were cultured, expanded and split. Replica plates were then subject to LacZ staining and PCR genotyping. Roughly, $30 \%$ of the colonies picked were LacZ negative and the initial $\mathrm{KO}$ allele was converted to a rWT allele, in which exon 8 of BAF250a is flanked by loxP sites (data not shown).

To target the second WT allele of BAF250a, the rWT $(+/ \mathrm{f})$ ES cells were electroporated a second time with the same BAF250a KO vector and selected with G418. Not surprisingly, due to preferential retargeting of the first modified allele, only about $10 \%$ of the colonies identified carried both targeted alleles (BAF250a -/f, Figure 1D), similar to what has been observed in our mouse BAF250a KO studies [9]. To avoid the possibility that constitutive Cre expression may have a detrimental effect on BAF250a KO ES cells, we next transfected a plasmid expressing the ligand-inducible Cre recombinase (CreERT2) and containing a puromycin cassette [12]. After puromycin selection, colonies were expanded and treated with $2.5 \mu \mathrm{M} 4-\mathrm{OH}$ TAM. The 4-OH TAM treatment induced deletions between the two loxP sites on each targeted allele to produce cells lacking both copies of exon 8 of BAF250a (Figure 1E). Removal of exon 8 with Cre recombinase is predicted to create a frameshift mutation and induce nonsense-mediated decay of the mutant transcript. Indeed, after $24 \mathrm{~h}$ of $4-\mathrm{OH}$ TAM treatment, the expression of BAF250a mRNA and protein dropped to background levels (Figure $1 \mathrm{~F}$ and $1 \mathrm{G}$ ).

We next examined the effects of BAF250a ablation on the self-renewal and differentiation of the H9 hES cells. At least three different KO hES cell lines were used for these assays. Quantitiative (q) RT-PCR (Supplementary information, Figure S1A) and immunostaining of pluri- potency markers Nanog and Oct4 (Figure 1H) showed that their expression was largely normal in BAF250a KO hES cells shortly after tamoxifen treatment. We also examined the apoptotic marker Casp3, which appeared not affected by BAF250a ablation, either (Supplementary information, Figure S1A). The BAF250a KO hES cells can be passaged for more than 10 times with rather normal morphology, and the morphologies of Het and KO hES cells appeared similar even under feeder-free culture conditions (Supplementary information, Figure S1B). Therefore, it appeared that BAF250a ablation did not lead to immediate differentiation as we observed in mouse BAF250a KO ES cells under feeder-free culturing conditions [9].

However, by examining the germ layer markers in ES cells and embryoid bodies (EBs), we did observe premature differentiation of the human BAF250a KO ES cells. Germ layer markers were prematurely expressed in these KO hES cells, and their expression was much higher in EBs from KO hES cells than from control Het cells (Supplementary information, Figure S1C). At the same time, a large percentage of EBs generated from $\mathrm{KO} h E S$ cells quickly turned into cystic-like EBs $(\sim 30 \%$ at Day 5 and $\sim 70 \%$ at Day 7 ), whereas the Het control EBs did not become cystic (Supplementary information, Figure S1D). These data suggest that like mouse ES cells, hES cells without BAF250a are prone to differentiation.

To test whether this KO strategy is applicable to other hES cell lines, the linearized BAF250a targeting vector was also electroporated into HUES8 hES cells, and three out of the four colonies that we picked were positively targeted BAF250a Het hES cell lines, suggesting that this strategy may work for many hES cell lines.

In this study, we report an efficient KO strategy to ablate gene function in hES cells. Using a promoterless KO strategy, we first converted a WT BAF250a allele into a mutant allele. Flpase-mediated recombination then converted the initial KO allele back into a WT allele. The same $\mathrm{KO}$ vector was applied the second time to mutate the second WT allele in hES cells. Finally, Cre-mediated recombination rendered both modified alleles into $\mathrm{KO}$ alleles (Figure 1B and $1 \mathrm{~F}$ ). This highly efficient and controlled gene KO strategy will be especially useful to study of essential genes in hES cells.

We envision that alternatives of this strategy can be applied to introduce point mutation, insertion or deletion of target genes, or knock-in genes of interest to the modified gene locus (Supplementary information, Figure S2A and S2B). A second selection gene (such as hygromycin, blasticidin etc.) could be used in the second allele targeting vector to reduce the manipulations and increase the overall efficiency of the process. Furthermore, the 
knock-in gene(s) can be further replaced efficiently by yet another gene(s) by Cre-mediated recombination and selection against G418, similar to a strategy described in mouse ES studies [13] (Supplementary information, Figure S2B).

The highly efficient homologous recombination in this strategy seems to be dependent on the expression of the target gene [10], although genes with non-detectable expression in mouse ES cells can be targeted fairly efficiently, too (William C Skarnes, personal communication). Thus, further work may be needed to establish whether tissue-specific genes can be targeted efficiently in hES cells.

This strategy can also be applied to human somatic cell lines. In fact, similar studies were performed in human cancer cells and other recombinogenic human cell lines $[14,15]$. We envision that alternatives of this strategy can be applied to introduce modifications in genes of interest to the modified gene locus (Supplementary information, Figure S2A and S2B). Our study, together with other strategies, will provide complementary and alternative approaches to manipulating gene activity in hES cells, and help identify transcription network in hES cells and establish hES cell-based disease models and therapies.

\section{Acknowledgments}

We thank Dr William C Skarnes at Wellcome Trust Sanger Institute for technical support. This work is supported by an MGH internal fund to ZW and grants from Harvard Stem Cell Institute To KRC and ZW.

Lei $\mathrm{Bu}^{1}$, Xiaolin $\mathrm{Gao}^{1}$, Xin Jiang ${ }^{1}$, Kenneth R Chien ${ }^{1}$, Zhong Wang ${ }^{1}$

${ }^{l}$ Cardiovascular Research Center, Massachusetts General Hospital, Harvard Medical School and Harvard Stem Cell Institute, Richard Simches Research Center, 185 Cambridge Street, Boston, MA 02114, USA

Correspondence: Kenneth R Chien ${ }^{\mathrm{a}}$, Zhong Wang ${ }^{\mathrm{b}}$

${ }^{a}$ Tel: 617-643-3440; Fax: 617-643-3445

E-mail:kchien@partners.org

${ }^{b}$ Tel: 617-643-3444; Fax: 617-643-3451

E-mail: zhwang@partners.org

\section{References}

1 Capecchi MR. Generating mice with targeted mutations. Nat
Med 2001; 7:1086-1090.

2 te Riele H, Maandag ER, Clarke A, Hooper M, Berns A. Consecutive inactivation of both alleles of the pim-1 protooncogene by homologous recombination in embryonic stem cells. Nature 1990; 348:649-651.

3 Mortensen RM, Conner DA, Chao S, Geisterfer-Lowrance AA, Seidman JG. Production of homozygous mutant ES cells with a single targeting construct. Mol Cell Biol 1992; 12:23912395.

4 Zwaka TP, Thomson JA. Homologous recombination in human embryonic stem cells. Nat Biotechnol 2003; 21:319-321.

5 Thyagarajan B, Liu Y, Shin S, et al. Creation of engineered human embryonic stem cell lines using phiC31 integrase. Stem Cells 2008; 26:119-126.

6 Lombardo A, Genovese P, Beausejour CM, et al. Gene editing in human stem cells using zinc finger nucleases and integrasedefective lentiviral vector delivery. Nat Biotechnol 2007; 25:1298-1306.

7 Zou J, Maeder ML, Mali P, et al. Gene targeting of a diseaserelated gene in human induced pluripotent stem and embryonic stem cells. Cell Stem Cell 2009; 5:97-110.

8 Hockemeyer D, Soldner F, Beard C, et al. Efficient targeting of expressed and silent genes in human ESCs and iPSCs using zinc-finger nucleases. Nat Biotechnol 2009; 27:851-857.

9 Gao X, Tate P, Hu P, Tjian R, Skarnes WC, Wang Z. ES cell pluripotency and germ layer formation require the SWI/SNF chromatin remodeling component BAF250a. Proc Natl Acad Sci USA 2008; 105:6656-6661.

10 Friedel RH, Plump A, Lu X, et al. Gene targeting using a promoterless gene trap vector ("targeted trapping") is an efficient method to mutate a large fraction of genes. Proc Natl Acad Sci USA 2005; 102:13188-13193.

11 Feil R, Wagner J, Metzger D, Chambon P. Regulation of Cre recombinase activity by mutated estrogen receptor ligandbinding domains. Biochem Biophys Res Commun 1997; 237:752-757.

$12 \mathrm{Bu} \mathrm{L}$, Jiang X, Martin-Puig S, et al. Human ISL1 heart progenitors generate diverse multipotent cardiovascular cell lineages. Nature 2009; 460:113-117.

13 Beard C, Hochedlinger K, Plath K, Wutz A, Jaenisch R. Efficient method to generate single-copy transgenic mice by sitespecific integration in embryonic stem cells. Genesis 2006; 44:23-28.

14 Adachi N, Kurosawa A, Koyama H. Highly proficient gene targeting by homologous recombination in the human pre-B cell line Nalm-6. Methods Mol Biol 2008; 435:17-29.

15 Chen T, Hevi S, Gay F, et al. Complete inactivation of DNMT1 leads to mitotic catastrophe in human cancer cells. Nat Genet 2007; 39:391-396.

(Supplementary information is linked to the online version of the paper on the Cell Research website.) 